\title{
ARTICLE
}

\section{A two-dimensional spin field-effect switch}

\author{
Wenjing Yan ${ }^{1, \star}$, Oihana Txoperena ${ }^{1, \star}$, Roger Llopis $^{1}$, Hanan Dery ${ }^{2,3}$, Luis E. Hueso ${ }^{1,4}$ \& Fèlix Casanova ${ }^{1,4}$
}

Future development in spintronic devices will require an advanced control of spin currents, for example by an electric field. Here we demonstrate an approach that differs from previous proposals such as the Datta and Das modulator, and that is based on a van de Waals heterostructure of atomically thin graphene and semiconducting $\mathrm{MoS}_{2}$. Our device combines the superior spin transport properties of graphene with the strong spin-orbit coupling of $\mathrm{MoS}_{2}$ and allows switching of the spin current in the graphene channel between ON and OFF states by tuning the spin absorption into the $\mathrm{MoS}_{2}$ with a gate electrode. Our proposal holds potential for technologically relevant applications such as search engines or pattern recognition circuits, and opens possibilities towards electrical injection of spins into transition metal dichalcogenides and alike materials.

\footnotetext{
${ }^{1} \mathrm{CIC}$ nanoGUNE, Tolosa Hiribidea 76, Donostia-San Sebastian, 20018 Basque Country, Spain. ${ }^{2}$ Department of Electrical and Computer Engineering, University of Rochester, Rochester, New York 14627, USA. ${ }^{3}$ Department of Physics and Astronomy, University of Rochester, Rochester, New York 14627, USA ${ }^{4}$ IKERBASQUE, Basque Foundation for Science, Bilbao, 48013 Basque Country, Spain. * These authors contributed equally to this work. Correspondence and requests for materials should be addressed to L.E.H. (email: I.hueso@nanogune.eu) or to F.C. (email: f.casanova@nanogune.eu).
} 
T he integration of the spin degree of freedom in chargebased electronic devices has revolutionized both sensing and memory capability in microelectronics ${ }^{1}$. However, for allowing further development and a successful implementation of spin logic circuits, an electrical manipulation of spin currents is required. The approach followed so far, inspired by the seminal proposal of the Datta and Das spin modulator ${ }^{2}$, has relied on the spin-orbit field as a medium for electrical control of the spin current $^{3-6}$. However, a challenge is to engineer a material that is capable of transporting spins over long distances and meanwhile has a strong enough spin-orbit coupling (SOC) to allow their electrical manipulation at temperatures above few Kelvin.

For example, carbon-based materials with intrinsic weak SOC, such as organic semiconductors ${ }^{7}$, carbon nanotubes ${ }^{8}$ and graphene ${ }^{9}$, have made a notable impact in spintronics. In particular, graphene has been proved to be ideal for long-distance spin transport (in excess of several micrometres) ${ }^{10-15}$. However, owing to its weak SOC, spin manipulation in this material has been mainly achieved by an external magnetic field through Hanle precession ${ }^{10,13,14}$. Although various approaches have been taken to enhance the SOC of graphene, for example through proximity effect ${ }^{16-18}$ or by atomic doping ${ }^{19}$, a direct evidence on the modulation of spin transport by an electric field remains elusive.

Meanwhile, transition metal dichalcogenides (TMDs) have emerged to complement graphene due to their unique optical, spin and valley properties ${ }^{20,21}$. Specifically, $\mathrm{MoS}_{2}$, the best-known member of that class, has a crossover from an indirect to a direct-gap semiconductor when thinned down to a monolayer $(\mathrm{ML})^{22}$. Its electronic properties can be strongly modulated by gate, large current ON/OFF ratio as much as $1 \times 10^{8}$ in ML and $1 \times 10^{6}$ in multilayers have been found $d^{23,24}$. Its stronger SOC compared with that of graphene, arising from the $d$-orbitals of the transition metal atoms, offers new possibilities to employ the spin and valley degrees of freedom in TMDs ${ }^{20,25-27}$.

In our work, the combination of graphene with $\mathrm{MoS}_{2}$ in a heterostructure through weak van der Waals (vdW) forces ${ }^{28}$ allows us to engineer an alternative type of field-effect switch for spin transport. A spin current in the graphene section of the device is electrically injected from a ferromagnetic source terminal. The gate electrode controls how much of that spin current is absorbed by the intersecting $\mathrm{MoS}_{2}$ layer (spin sink) before its arrival to the ferromagnetic drain terminal. By tuning the gate voltage, we were able to switch the spin current between binary ON and OFF states at temperatures up to $200 \mathrm{~K}$. The current device could be scalable and operative at room temperature, considering both the rapid progress made in chemical vapour deposition of two-dimensional (2D) materials and the theoretical performance of the materials involved.

\section{Results}

Device structure and measurement configurations. A sketch of the $2 \mathrm{D} v \mathrm{vdW}$ heterostructure and the electrical measurement scheme is shown in Fig. 1a, whereas a scanning electron microscope image of the device is shown in Fig. 1b. Graphene flakes are exfoliated onto a highly doped Si substrate covered by $300 \mathrm{~nm}$ of $\mathrm{SiO}_{2}$. An ML graphene flake is identified according to its optical contrast ${ }^{29}$ and, subsequently, a few-layer $\mathrm{MoS}_{2}$ flake is transferred above it by all-dry viscoelastic stamping ${ }^{30}$. Several $\mathrm{Co} / \mathrm{TiO}_{2}$ electrodes are patterned by electron-beam lithography and evaporated onto the graphene channel, to create lateral spin valves (LSVs; see Methods), which enable the injection and detection of pure spin currents in graphene in a non-local geometry ${ }^{10,14}$. The non-local resistance $R_{\mathrm{nl}}=V_{\mathrm{nl}} / I$, which depends on the relative orientation of the magnetisation of the injecting and detecting Co electrodes, is measured while sweeping the magnetic field $\mathbf{B}$ in-plane along the easy axis of the electrodes (see Fig. 1a for a sketch of the experimental geometry). Specifically, when the configuration of the magnetizations changes from parallel to antiparallel, $R_{\mathrm{n} 1}$ switches from high $\left(R_{\mathrm{p}}\right)$ to low $\left(R_{\mathrm{ap}}\right)$ value. The spin signal is proportional to the amount of spin current reaching the detector, measured by $\Delta R_{\mathrm{nl}}=R_{\mathrm{p}}-R_{\mathrm{ap}}$ (Fig. 1c).

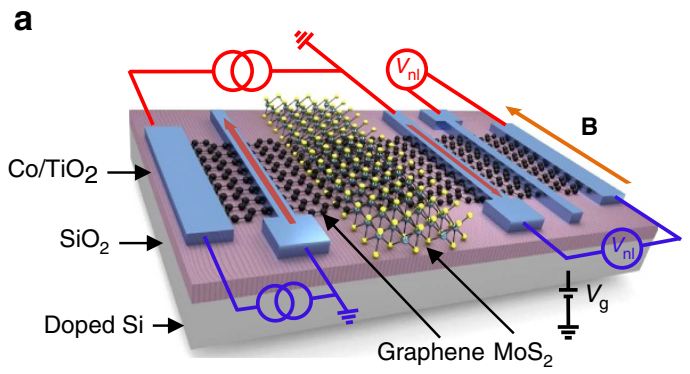

C
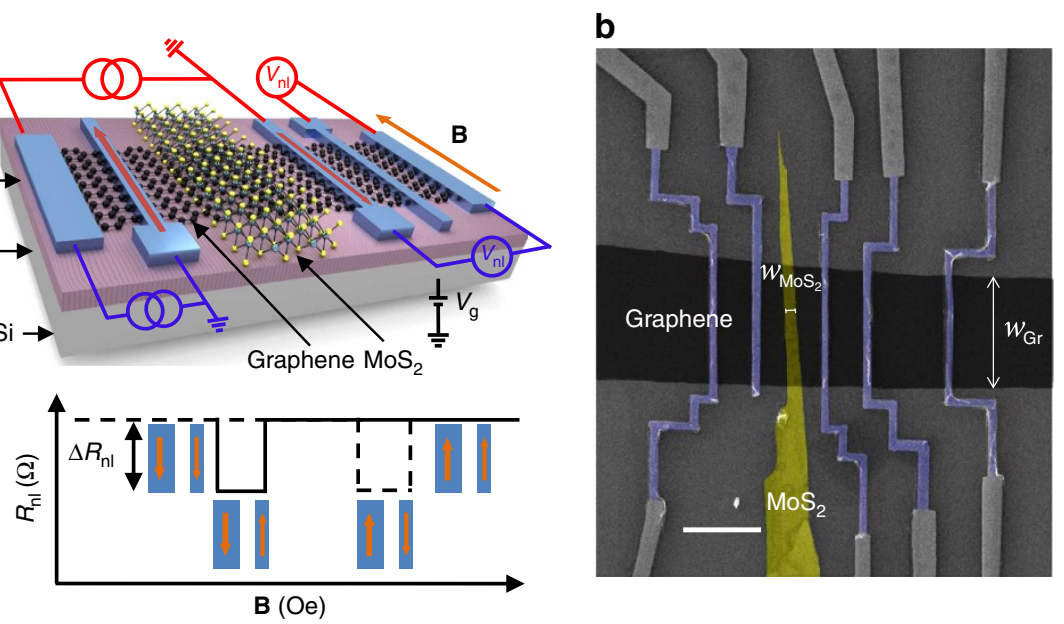

Figure 1 | Illustration of the experiment and scanning electron microscope (SEM) image of the device. (a) Sketch of the 2D vdW heterostructure to be used for switching the spin transport. For the non-local measurement, a DC current $(10 \mu \mathrm{A})$ is injected into graphene from a ferromagnetic Co electrode across a $\mathrm{TiO}_{2}$ barrier and a non-local voltage $\left(V_{\mathrm{nl}}\right)$ is measured by a second Co electrode while sweeping the magnetic field $\mathbf{B}$. The red- and blue-coloured circuit diagrams represent the measurement configurations in the reference graphene LSV (without $\mathrm{MoS}_{2}$ on top) and the graphene/MoS 2 LSV (with MoS 2 intercepting the spin current path). In the latter case, the spin current flowing in the graphene can be switched ON and OFF by modulating the conductivity of $\mathrm{MoS}_{2}$ using an electric field across a $\mathrm{SiO}_{2}$ dielectric (also shown in the diagram). (b) False-coloured SEM image of the LSV devices. The width of the graphene and $\mathrm{MoS}_{2}$ are $w_{\mathrm{Gr}}=3 \mu \mathrm{m}$ and $w_{\mathrm{MoS}_{2}}=0.4 \mu \mathrm{m}$, respectively. Scale bar, $2 \mu \mathrm{m}$. (c) An illustration of a typical non-local magnetoresistance measurement, where the non-local resistance $R_{\mathrm{nl}}$ switches between $R_{\mathrm{P}}$ and $R_{\mathrm{AP}}$ for parallel and antiparallel magnetization orientations of the Co electrodes. The spin signal is tagged as $\Delta R_{\mathrm{nl}}=R_{\mathrm{p}}-R_{\mathrm{ap}}$. 

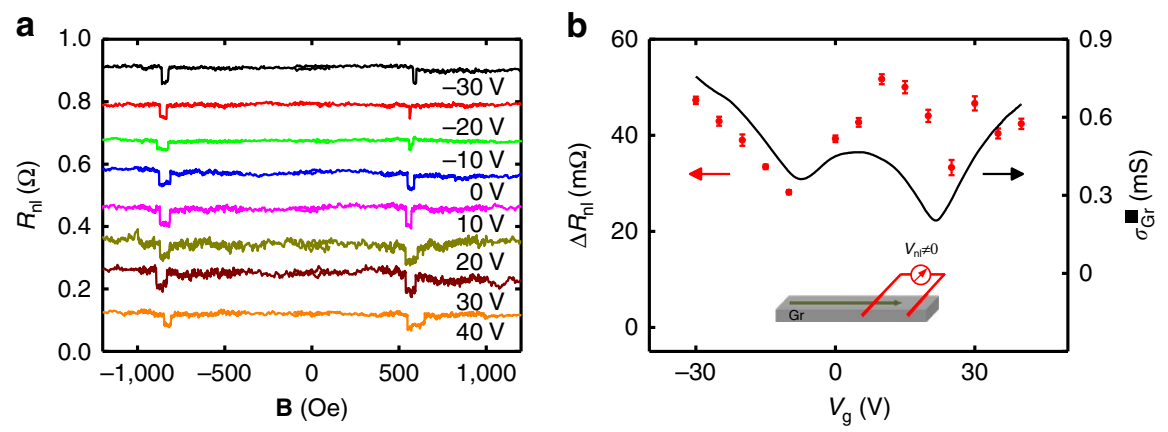

Figure 2 | Spin transport in a reference graphene lateral spin valve. Measurements are done using the red-coloured circuit diagram in Fig. 1a. (a) Non-local resistance $R_{\mathrm{nl}}$ as a function of the magnetic field $\mathbf{B}$ measured at different $V_{\mathrm{g}}$ at $50 \mathrm{~K}$. The current bias is $10 \mu \mathrm{A}$ and the centre-to-centre distance between ferromagnetic electrodes $(L)$ is $1 \mu \mathrm{m}$. Individual sweeps are offset in $R_{\mathrm{nl}}$ for clarity. (b) Spin signal $\Delta R_{\mathrm{nl}}$ measured at different $V_{\mathrm{g}}$ (red circles). The black solid line shows the sheet conductivity of the graphene as a function of $V_{\mathrm{g}}$. The inset shows schematically the spin current (green arrow) reaching the detector in the full range of $V_{g}$. Error bars are calculated using the s.e. associated with the statistical average of the non-local resistance in the parallel and antiparallel states.

Spin transport in a reference graphene lateral spin valve. We first study the spin transport in a graphene LSV without $\mathrm{MoS}_{2}$ (reference LSV). Figure 2a shows the measured $R_{\mathrm{nl}}$ as a function of $\mathbf{B}$ for different gate voltages $\left(V_{\mathrm{g}}\right)$. On application of $V_{\mathrm{g}}$, the magnitude of the spin signal weakly varies, following the modification of the graphene sheet conductivity $\left(\sigma_{\mathrm{Gr}}\right)$ with $V_{\mathrm{g}}$, as can be observed in Fig. 2b. The correlation between $\Delta R_{\mathrm{nl}}$ and $\sigma_{\mathrm{Gr}}^{\mathbf{n}}$ is a signature of a transparent interface between the $\mathrm{Co} / \mathrm{TiO}_{2}$ electrodes and the graphene $(\sim 250 \Omega)$, as it is well established in the literature ${ }^{14}$.

Spin transport in a graphene/ $\mathrm{MoS}_{2}$ spin field-effect switch. Next, we introduce the central results of our manuscript: the demonstration of spin switching by a gate voltage in a graphene/ $\mathrm{MoS}_{2}$ LSV. Figure $3 \mathrm{a}$ shows $R_{\mathrm{nl}}$ of this device, while sweeping B for different values of $V_{\mathrm{g}}$, where a gradual decrease of the spin signal $\Delta R_{\mathrm{nl}}$ with $V_{\mathrm{g}}$ can be observed. This behaviour is clearly seen in Fig. 3b, where $\Delta R_{\mathrm{nl}}$ is plotted as a function of $V_{\mathrm{g}}$, showing the decay of $\Delta R_{\mathrm{nl}}$ towards zero at positive values of $V_{\mathrm{g}}$, in contrast with the weakly varying spin signal measured in the reference LSV (see Fig. 2b). Figure $3 \mathrm{~b}$ also plots the $\mathrm{MoS}_{2}$ sheet conductivity $\left(\sigma_{\mathrm{MoS}_{2}}^{\mathbf{g}}\right)$ from a reference device revealing an opposite gate voltage dependence to that of $\Delta R_{\mathrm{nl}}$. For large negative $V_{\mathrm{g}}$, the semiconducting $\mathrm{MoS}_{2}$ is in the low conductivity OFF state and the measured $\Delta R_{\mathrm{nl}}$ value is comparable to that of the reference LSV, reaching the ON state of the device. This result is expected, considering that the electrode spacing here is slightly longer than in the reference LSV $(1.8$ versus $1 \mu \mathrm{m}$; see Fig. $1 \mathrm{~b}$ for comparison). Sweeping the gate voltage towards positive values brings the $\mathrm{MoS}_{2}$ towards its high conductivity ON state, where $\sigma_{\mathrm{MoS}_{2}}$ increases by more than six orders of magnitude compared with the OFF state. Simultaneously, the spin current reaching the detector and the corresponding $\Delta R_{\mathrm{nl}}$ gradually decrease towards zero (see Fig. 3b), reaching the OFF state of the device for $V_{\mathrm{g}}>15 \mathrm{~V}$. The change in spin signal per gate voltage unit in our device is $\sim 0.7 \mathrm{~m} \Omega \mathrm{V}^{-1}$. The results are completely reproducible upon multiple gate voltage sweeps and temperature cycles, evidencing the robustness of the effect (Supplementary Fig. 1). Similar results to those in Fig. $3 \mathrm{~b}$ are also observed at temperatures up to $200 \mathrm{~K}$ (Supplementary Fig. 2).

This control of the spin current directly demonstrates the $2 \mathrm{D}$ spin field-effect switch. This proof-of-principle effect can be enhanced by using a different dielectric, for example, layered hexagonal $\mathrm{BN}^{24,31}$, or by tuning the interface resistance between graphene and $\mathrm{Co}^{14}$.

\section{Discussion}

The switching of spin transport using the graphene/ $\mathrm{MoS}_{2} \mathrm{vdW}$ heterostructure relies on the absorption of spins travelling through the graphene by the $\mathrm{MoS}_{2}$, as schematically illustrated in the inset of Fig. 3b. To support this argument, we make use of the spin resistances of the channel (graphene) and the absorbing material $\left(\mathrm{MoS}_{2}\right)$, which are the main control parameters in the spin absorption mechanism. Roughly, they can quantify how easily the spin current flows through each of the materials, in the same way in which one can estimate a charge current flow in parallel electrical resistors. The spin resistances of graphene and $\mathrm{MoS}_{2}$ can be expressed as $R_{\mathrm{Gr}}^{\mathrm{S}}=\frac{R_{\mathrm{Gr}}^{\mathbf{m}_{\mathrm{Gr}}} \lambda_{\mathrm{Gr}}}{w_{\mathrm{Gr}}}$ and $R_{\mathrm{MoS}_{2}}^{\mathrm{S}}=\frac{R_{\mathrm{Mos}_{2}}^{\boldsymbol{m}_{2}}\left(\lambda_{\mathrm{Mos}_{2}}\right)^{2}}{w_{\mathrm{Gr}} w_{\mathrm{Mos}_{2}}}$, respectively (Supplementary Note 2); where $R_{\mathrm{Gr}, \mathrm{MoS}_{2}}^{\mathbf{m}}=1 / \sigma_{\mathrm{Gr}, \mathrm{MoS}_{2}}^{\mathbf{m}}$ are their sheet resistances, $\lambda_{\mathrm{Gr}, \mathrm{MoS}_{2}}$ their spin diffusion lengths and $w_{\mathrm{Gr}, \mathrm{MoS}_{2}}$ their widths $\left(w_{\mathrm{Gr}}=3 \mu \mathrm{m}\right.$ and $\left.w_{\mathrm{MoS}_{2}}=0.4 \mu \mathrm{m}\right)$. We have estimated the intrinsic spin lifetime in bulk $\mathrm{MoS}_{2}$ to be in the range of $10 \mathrm{ps}$ (see Supplementary Note 2). For this estimation, we have considered electron interaction with flexural phonons and found weak temperature dependence of the spin relaxation in accord with our experimental results and in contrast to the findings in $\mathrm{ML} \mathrm{MoS}$ (ref. 27). The lack of space inversion symmetry in a ML has two effects on the spin transport. The first one is to increase the amplitude of the spin-flip matrix element ${ }^{26}$. The second effect is to induce spin splitting of the energy bands at the $K$ point. Although the former effect enhances spin relaxation, the latter one suppresses it when the spin splitting is large enough to exclude elastic scattering. In $p$-type ML TMDs, for example, the spin splitting in the valence band is of the order of hundreds of $\mathrm{meV}$ and the overall spin lifetime is prolonged compared with bulk. In this view, the spin degeneracy of the energy bands in few-layer TMDs renders these materials ideal spin sinks when put in proximity to graphene. Using the estimated spin lifetime in bulk $\mathrm{MoS}_{2}$, we calculate $\lambda_{\mathrm{MoS}_{2}} \sim 20 \mathrm{~nm}$ in the OFF state of the device at $V_{\mathrm{g}}=40 \mathrm{~V}$. In contrast, the spin diffusion length in graphene is much longer, being $\lambda_{\mathrm{Gr}} \sim 1.2 \mu \mathrm{m}$ estimated from Hanle measurements on a reference device (Supplementary Fig. 3). Substituting the spin diffusion lengths, the electrical properties and geometrical factors of graphene and $\mathrm{MoS}_{2}$, we obtain $R_{\mathrm{Gr}}^{\mathrm{S}}=408 \Omega$ and $R_{\mathrm{MoS}_{2}}^{\mathrm{S}}=2.7 \Omega \quad V_{\mathrm{g}}=40 \mathrm{~V} \quad$ (Supplementary Note 2). The fact that $R_{\mathrm{MoS}_{2}}^{\mathrm{S}}<<R_{\mathrm{Gr}}^{\mathrm{S}}$ demonstrates the capability of the $\mathrm{MoS}_{2}$ to absorb spins from the graphene channel. This is further supported by the low graphene/ $\mathrm{MoS}_{2}$ barrier height at high positive $V_{\mathrm{g}}$ (ref. 32), making the interface resistance sufficiently low for efficient spin absorption. 

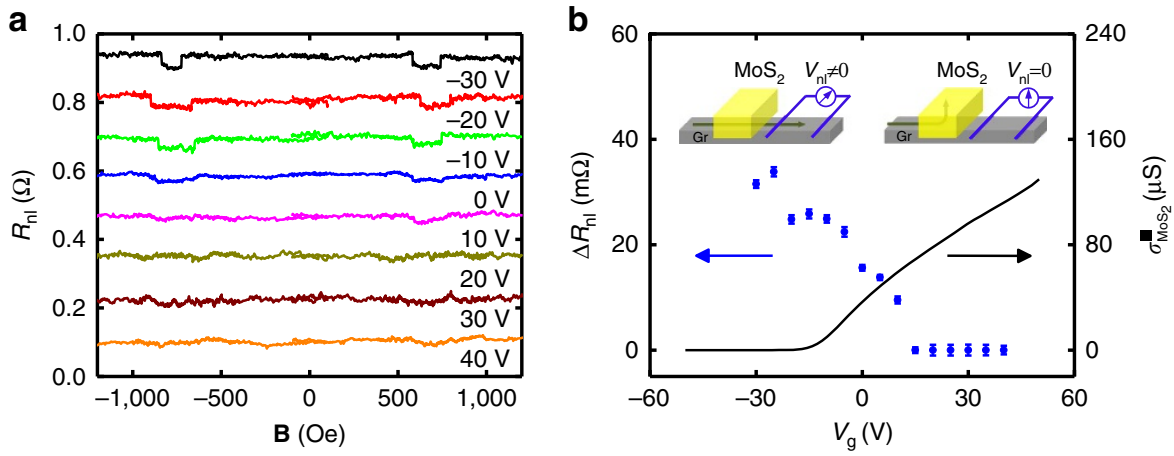

Figure 3 | Spin transport in a graphene/MoS $\mathbf{2}$ lateral spin valve. Measurements are done using the blue-coloured circuit diagram in Fig. 1a. (a) Non-local resistance $R_{\mathrm{nl}}$ measured as a function of the magnetic field $\mathbf{B}$ at different $V_{\mathrm{g}}$ at $50 \mathrm{~K}$ using $10 \mu \mathrm{A}$ current bias and for a centre-to-centre distance between ferromagnetic electrodes $(L)$ of $1.8 \mu \mathrm{m}$. Individual sweeps are offset in $R_{\mathrm{nl}}$ for clarity. (b) Gate modulation of the spin signal $\Delta R_{\mathrm{nl}}$ (blue circles). The black solid line is the sheet conductivity of the $\mathrm{MoS}_{2}$ as a function of $V_{\mathrm{g}}$. The insets show schematically the spin current path (green arrow) in the OFF state (left inset) and the ON state (right inset) of $\mathrm{MoS}_{2}$. Error bars are calculated using the s.e. associated with the statistical average of the nonlocal resistance in the parallel and antiparallel states.

The situation completely changes when the gate voltage $V_{\mathrm{g}}$ is swept towards negative values. At $V_{\mathrm{g}}=-30 \mathrm{~V}$, the $\mathrm{MoS}_{2}$ conductivity $\sigma_{\mathrm{Mos} /}$ decreases by more than six orders of magnitude from $V_{\mathrm{g}}=40 \mathrm{~V}$, which leads to a similar increase in $R_{\mathrm{MoS}_{2}}^{\mathrm{S}}$. Therefore, we have $R_{\mathrm{MoS}_{2}}^{\mathrm{S}}>>R_{\mathrm{Gr}}^{\mathrm{S}}$, preventing spin absorption by $\mathrm{MoS}_{2}$. Although the very large spin resistance of $\mathrm{MoS}_{2}$ alone is sufficient to support the vanishing spin absorption, we note that the interface resistance between graphene and $\mathrm{MoS}_{2}$ also increases ${ }^{32}$, further preventing probable spin absorption into the $\mathrm{MoS}_{2}$. Therefore, the gate dependence of the graphene/MoS interface resistance acts as a positive feedback, further improving the performance in the regime between the fully ON and OFF state of the device.

The inverse correlation between the spin signal $\Delta R_{\mathrm{nl}}$ and the $\mathrm{MoS}_{2}$ conductivity $\sigma_{\mathrm{MoS}_{2}}$ can be clearly seen in Fig. 3b. This correlation supports the aforementioned argument and discards other scenarios, such as spin dephasing in possible trap states at the graphene/ $\mathrm{MoS}_{2}$ interface. The fact that similar results to those in Fig. $3 \mathrm{~b}$ are also observed at $200 \mathrm{~K}$ indicates that the effect barely changes with temperature and therefore is incompatible with the exponential temperature dependence expected for capture and escape in trap states (Supplementary Note 1). Next, we confirm the spin absorption mechanism by computing the expected spin signal ratio, $\Delta R_{\mathrm{nl}}^{\text {abs }} / \Delta R_{\mathrm{nl}}$, which quantifies the relative amount of spins deviating from the graphene channel towards the $\mathrm{MoS}_{2}$ (ref. 33):

$$
\frac{\Delta R_{\mathrm{nl}}^{\mathrm{abs}}}{\Delta R_{\mathrm{nl}}}=
$$

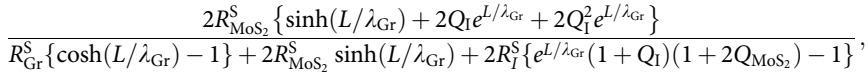

where $\Delta R_{\mathrm{nl}}^{\mathrm{abs}}$ and $\Delta R_{\mathrm{nl}}$ are the spin signals with and without spin absorption by the $\mathrm{MoS}_{2} ; R_{\mathrm{I}}^{\mathrm{S}}=R_{\mathrm{I}} /\left(1-P_{\mathrm{I}}^{2}\right)$ is the spin resistance of the $\mathrm{Co} / \mathrm{TiO}_{2} /$ graphene interface, $R_{\mathrm{I}}$ is the interface resistance and $P_{\mathrm{I}}$ is the interface spin polarisation; and finally, $Q_{\mathrm{Mos}_{2}}=\frac{R_{\mathrm{Mos}}^{\mathrm{s}}}{R_{\mathrm{Gr}}^{\mathrm{s}}}$ and $Q_{\mathrm{I}}=\frac{R_{\mathrm{I}}^{\mathrm{S}}}{R_{\mathrm{Gr}}^{\mathrm{S}}}$. Assuming the interface is transparent at $40 \mathrm{~V}$, one can calculate the expected spin signal ratio when the $\mathrm{MoS}_{2}$ is fully ON. Substituting the known parameters into equation (1), one gets $\Delta R_{\mathrm{nl}}^{\text {abs }} / \Delta R_{\mathrm{nl}} \approx 0.017$ at $V_{\mathrm{g}}=40 \mathrm{~V}$ (Supplementary Note 3).
The very small value calculated for $\Delta R_{\mathrm{nl}}^{\mathrm{abs}} / \Delta R_{\mathrm{nl}}$ predicts a strong spin absorption, which confirms this scenario to be responsible for the observed experimental results of Fig. 3b.

Compared with previous Datta and Das-like spin modulators ${ }^{3-6}$, the electrical manipulation of spin transport in our 2D spin fieldeffect switch is observed at much higher temperature (up to $200 \mathrm{~K}$ versus few or sub $\mathrm{K}$ ). It also displays well-defined $\mathrm{ON}$ and OFF states, which are easily controlled by the gate electric field instead of an oscillatory spin signal. Moreover, there is plenty of room for the optimization of the device performance. For instance, by incorporating tunnel barriers with higher resistance, the spin signal and thus the difference between the $\mathrm{ON}$ and $\mathrm{OFF}$ states could be increased by two orders of magnitude ${ }^{34-36}$. The threshold voltage required to turn $\mathrm{ON}$ and OFF the device can be reduced by replacing $\mathrm{SiO}_{2}$ with a thinner dielectric of larger dielectric constant, such as $\mathrm{HfO}_{2}$ (ref. 37) or hexagonal $\mathrm{BN}^{24,31}$. With the above improvements to the fabrication process, and considering the robust performance of $\mathrm{MoS}_{2}$ transistors at room temperature ${ }^{23,24}$, a room-temperature $2 \mathrm{D}$ spin field-effect switch is envisioned. The recent advances in chemical growth of high quality $2 \mathrm{D}$ layered materials ${ }^{21,38}$ and their heterostructure multilayers ${ }^{39-41}$, as well as in homostructural ${ }^{42}$, and heterostructural $^{36}$ tunnel barriers for spin injection, may well lead to large-scale integration of the current device architecture. Aside from the potential technological applications, the spin absorption effect in our experiments provides a solution to electrically inject spins into 2D semiconducting TMDs, which has so far been elusive due to the conductivity mismatch problem ${ }^{43-45}$.

In conclusion, the seamless integration of two 2D layered materials with remarkably different spin-orbit coupling amplitudes leads to a device capable of both transporting and electrically controlling a spin current. The demonstrated $2 \mathrm{D}$ spin field-effect switch can improve the performance of search engines or pattern recognition circuits, wherein a large number of independent logic operations are executed in parallel ${ }^{15,46,47}$. Furthermore, the vdW heterostructure at the core of our experiments opens the path for fundamental research of exotic transport properties predicted for $\mathrm{TMDs}^{20,25,26}$.

\section{Methods}

Device fabrication. Fabrication of ML graphene samples uses the mechanical exfoliation method initiated in ref. 29. We first exfoliate bulk graphitic crystals onto a Nitto tape (Nitto SPV 224P) and repeat the cleavage process between three and 
five times until thin flakes can be identified visually by the eye. The Nitto tape with relatively thin flakes is pressed against a preheated $\mathrm{Si}$ substrate with $300 \mathrm{~nm} \mathrm{SiO}$. After peeling off the Nitto tape, the substrate is examined under an optical microscope and ML graphene is identified by well-established optical contrast. We then prepare the $\mathrm{MoS}_{2}$ /poly-dimethyl siloxane stamp following ref. 30. First, a $\mathrm{MoS}_{2}$ crystal is exfoliated twice using the Nitto tape and transferred on to a piece of poly-dimethyl siloxane (Gelpak PF GEL film WF $\times 4,17$ mil.). After identifying the desired few-layer $\mathrm{MoS}_{2}$ flake using optical contrast, it is transferred on top of graphene after slowly removing the viscoelastic stamp.

The lateral spin valve is formed following a standard nanofabrication procedure including electron-beam lithography, metal deposition and metal lift-off in acetone. $5 \AA$ of Ti are deposited by electron-beam evaporation and left to oxidize in air for $0.5 \mathrm{~h}$ before depositing $35 \mathrm{~nm}$ of Co using electron-beam evaporation.

Electrical measurements. The measurements are performed in a Physical Property Measurement System by Quantum Design, using a 'DC reversal' technique with a Keithley 2182 nanovoltmeter and a 6221 current source. A current bias of $10 \mu \mathrm{A}$ is used unless stated in the text. Gate voltage is applied using a Keithley model 2636. The gate voltage is applied between the back of the doped Si substrate and the grounding electrode. As graphene layer on the bottom is ML, it does not fully screen the gate electric field. Therefore, the gate voltage modulates the charge carrier density in both graphene and the $\mathrm{MoS}_{2}$

Data availability. All relevant data are available from the authors.

\section{References}

1. Fert, A. Nobel lecture: origin, development, and future of spintronics. Rev. Mod. Phys. 80, 1517-1530 (2008).

2. Datta, S. \& Das, B. Electronic analog of the electro-optic modulator. Appl. Phys. Lett. 56, 665-667 (1990).

3. Koo, H. C. et al. Control of spin precession in a spin-injected field effect transistor. Science 325, 1515-1518 (2009).

4. Chuang, P. et al. All-electric all-semiconductor spin field-effect transistors. Nat. Nanotechnol. 10, 35-39 (2015).

5. Wunderlich, J. et al. Spin Hall effect transistor. Science 330, 1801-1804 (2010).

6. Choi, W. Y. et al. Electrical detection of coherent spin precession using the ballistic intrinsic spin Hall effect. Nat. Nanotechnol. 10, 666-670 (2015).

7. Dediu, A. V., Hueso, L. E., Bergenti, I. \& Taliani, C. Spin routes in organic semiconductors. Nat. Mater. 8, 707-716 (2009).

8. Hueso, L. E. et al. Transformation of spin information into large electrical signals using carbon nanotubes. Nature 445, 410-413 (2007).

9. Pesin, D. \& MacDonald, A. H. Spintronics and pseudospintronics in graphene and topological insulators. Nat. Mater. 11, 409-416 (2012).

10. Tombros, N. et al. Electronic spin transport and spin precession in single graphene layers at room temperature. Nature 448, 571-574 (2007).

11. Yan, W. et al. Long spin diffusion length in few-layer graphene flakes. Phys. Rev. Lett. 117, 147201 (2016).

12. Dlubak, B. et al. Highy efficient spin transport in epitaxial graphene on SiC. Nat. Phys. 8, 557-561 (2012).

13. Han, W., Kawakami, R. K., Gmitra, M. \& Fabian, J. Graphene spintronics. Nat. Nanotechnol. 9, 794-807 (2014).

14. Han, W. et al. Tunneling spin injection into single layer graphene. Phys. Rev. Lett. 105, 167202 (2010)

15. Wen, H. et al. Experimental demonstration of XOR operation in graphene magnetologic gates at room temperature. Phys. Rev. Appl. 5, 044003 (2016).

16. Wang, Z. et al. Strong interface-induced spin-orbit coupling in graphene on $\mathrm{WS}_{2}$. Nat. Commun. 6, 8339 (2015).

17. Calleja, F. et al. Spatical variation of a giant spin-orbit effect induces electron confinement in graphene on $\mathrm{Pb}$ islands. Nat. Phys. 11, 43-47 (2015).

18. Avsar, A. et al. Spin-orbit proximity effect in graphene. Nat. Commun. 5, 4875 (2014).

19. Balakrishnan, J., Koon, G. K. W., Jaiswall, M., Castro Neto, A. H. \& Özyilmaz, B. Colossal enhancement of the spin-orbit coupling in weakly hydrogenated graphene. Nat. Phys. 9, 284-287 (2013).

20. Xu, X., Yao, W., Xiao, D. \& Heinz, T. F. Spin and pseudospins in layered transition metal dichalcogenides. Nat. Phys. 10, 343-350 (2014).

21. Wang, Q. H., Kalantar-Zadeh, K., Kis, A., Coleman, J. N. \& Strano, M. S. Electronics and optoelectronics of two-dimensional transition metal dichalcogenides. Nat. Nanotechnol. 7, 699-712 (2012).

22. Mak, K. F., Lee, C., Hone, J., Shan, J. \& Heinz, T. F. Atomically thin $\mathrm{MoS}_{2}$ : a new direct-gap semiconductor. Phys. Rev. Lett. 105, 136805 (2010).

23. Radisavljevic, B., Radenovic, A., Brivio, J., Giacometti, V. \& Kis, A. Single-layer $\mathrm{MoS}_{2}$ transistors. Nat. Nanotechnol. 6, 147-150 (2011).

24. Txoperena, O. Spin injection in two-dimensional layered materials. $\mathrm{PhD}$ thesis, University of the Basque Country, Spain. http://www.nanogune.eu/publications/ p-1-phd-theses/2016?idThesis=73 (2016).
25. Xiao, D., Liu, G.-B., Feng, W., Xu, X. \& Yao, W. Coupled spin and valley physics in monolayers of $\mathrm{MoS}_{2}$ and other group-VI dichalcogenides. Phys. Rev. Lett. 108, 196802 (2012).

26. Song, Y. \& Dery, H. Transport theory of monolayer transition-metal dichalcogenides through symmetry. Phys. Rev. Lett. 111, 026601 (2013).

27. Yang, L. et al. Long-lived nanosecond spin relaxation and spin coherence of electrons in monolayer $\mathrm{MoS}_{2}$ and $\mathrm{WS}_{2}$. Nat. Phys. 11, 830-834 (2015).

28. Geim, A. K. \& Grigorieva, I. V. Van der Waals heterostructures. Nature 449, 419-425 (2013).

29. Novoselov, K. S. et al. Electric field effect in atomically thin carbon films. Science 306, 666-669 (2004).

30. Castellanos-Gomez, A. et al. Deterministic transfer of two-dimensional materials by all-dry viscoelastic stamping. 2D Mater. 1, 011002 (2014).

31. Lee, G-H. et al. Flexible and transparent $\mathrm{MoS}_{2}$ field-effect transistors on hexagonal Boron Nitride-graphene Heterostructures. ACS Nano 7, 7931-7936 (2013).

32. Cui, X. et al. Multi-terminal transport measurements of $\mathrm{MoS}_{2}$ using a van der Waals heterostructure device platform. Nat. Nanotechnol. 10, 534-540 (2015).

33. Niimi, Y. et al. Giant spin Hall induced by skew scattering from Bismuth impurities inside thin film CuBi alloys. Phys. Rev. Lett. 109, 156602 (2012).

34. Drögeler, M. et al. Spin lifetimes exceeding $12 \mathrm{~ns}$ in graphene nonlocal spin valve devices. Nano Lett. 16, 3533-3539 (2016).

35. Ingla-Aynés, J., Meijerink, R. J. \& van Wees, B. J. Eighty-eight percent directional guiding of spin currents with $90 \mu \mathrm{m}$ relaxation length in bilayer graphene using carrier drift. Nano Lett. 16, 4825-4830 (2016).

36. Kamalakar, M. V. et al. Enhanced tunnel spin injection into graphene using chemical vapour deposited hexagonal boron nitride. Sci. Rep. 4, 6146 (2014).

37. Kar, S. High Permittivity Gate Dielectric Materials (Springer, 2013).

38. Kamalakar, M. V., Groenveld, C., Dankert, A. \& Dash, S. P. Long distance spin communication in chemical vapour deposited graphene. Nat. Commun. 6, 6766 (2015).

39. Liu, Z. et al. Direct growth of graphene/hexagonal Boron Nitride stacked layers. Nano Lett. 11, 2032-2037 (2011).

40. Zhang, C. et al. Direct growth of large-area graphene and boron nitride heterostructures by a co-segregation method. Nat. Commun. 6, 6519 (2015).

41. McCreary, K. M. et al. Large-area synthesis of continuous and uniform $\mathrm{MoS}_{2}$ monolayer films on graphene. Adv. Funct. Mater. 24, 6449-6454 (2014).

42. Friedman, A. L. et al. Homoepitaxial tunnel barriers with functionalized graphene-on-graphene for charge and spin transport. Nat. Commun. 5, 3161 (2014).

43. Chen, J.-R. et al. Control of schottky barriers in single layer $\mathrm{MoS}_{2}$ transistors with ferromagnetic contacts. Nano Lett. 13, 3106-3110 (2013).

44. Dankert, A., Langouche, L., Kamalakar, M. V. \& Dash, S. P. High-performance molybdenum disulfide field-effect transistors with spin tunnel contacts. ACS Nano 8, 476-482 (2014).

45. Wang, W. et al. Controllable schottky barriers between $\mathrm{MoS}_{2}$ and Permalloy. Sci. Rep. 4, 6928 (2014).

46. Dery, H. et al. Nanospintronics based on magnetologic gates. IEEE Trans. Electron Devices 59, 259-262 (2012).

47. Wang, W. \& Jiang, Z. Magnetic content addressable memory. IEEE Trans. Mag. 43, 2355-2357 (2007).

\section{Acknowledgements}

The work at CIC nanoGUNE was supported by the European Union 7th Framework Programme under the Marie Curie Actions (607904-13-SPINOGRAPH) and the European Research Council (257654-SPINTROS), by the Spanish MINECO under Project Number MAT2015-65159-R and by the Basque Government under Project Number PC2015-1-01. The work at the University of Rochester was supported by the Department of Energy under Contract Number DE-SC0014349, National Science Foundation under Contract Number DMR-1503601 and the Defense Threat Reduction Agency under Contract Number HDTRA1-13-1-0013.

\section{Author contributions}

F.C. conceived the study. W.Y., O.T. and R.L. performed the experiments. W.Y, O.T., L.E.H. and F.C. and analysed the data, discussed the experiments and wrote the manuscript. H.D. performed theoretical calculations on the spin relaxation properties of $\mathrm{MoS}_{2}$. All the authors contributed to the scientific discussion and manuscript revision. L.E.H. and F.C. co-supervised the work.

\section{Additional information}

Supplementary Information accompanies this paper at http://www.nature.com/ naturecommunications 
Competing financial interests: The authors declare no competing financial interests.

Reprints and permission information is available online at http://npg.nature.com/ reprintsandpermissions/

How to cite this article: Yan, W. et al. A two-dimensional spin field-effect switch. Nat. Commun. 7, 13372 doi: 10.1038/ncomms13372 (2016).

Publisher's note: Springer Nature remains neutral with regard to jurisdictional claims in published maps and institutional affiliations. (c) (i) This work is licensed under a Creative Commons Attribution 4.0 International License. The images or other third party material in this article are included in the article's Creative Commons license, unless indicated otherwise in the credit line; if the material is not included under the Creative Commons license, users will need to obtain permission from the license holder to reproduce the material. To view a copy of this license, visit http://creativecommons.org/licenses/by/4.0/

(C) The Author(s) 2016 